\title{
REVIEW
}

\section{Diagnosis and management of somatosensory tinnitus: review article}

\author{
Tanit Ganz Sanchez, Carina Bezerra Rocha \\ Department of Otolaryngology, Faculdade de Medicina da Universidade de São Paulo, São Paulo/SP, Brazil.
}

Tinnitus is the perception of sound in the absence of an acoustic external stimulus. It affects 10-17\% of the world's population and it a complex symptom with multiple causes, which is influenced by pathways other than the auditory one. Recently, it has been observed that tinnitus may be provoked or modulated by stimulation arising from the somatosensorial system, as well as from the somatomotor and visual-motor systems. This specific subgroup - somatosensory tinnitus - is present in $65 \%$ of cases, even though it tends to be underdiagnosed. As a consequence, it is necessary to establish evaluation protocols and specific treatments focusing on both the auditory pathway and the musculoskeletal system.

KEYWORDS: auditory pathway; ear; neuroplasticity; myofascial pain syndrome; musculoskeletal.

Sanchez TG, Rocha CB. Diagnosis and management of somatosensory tinnitus: review article. Clinics. 2011;66(6):1089-1094.

Received for publication on January 16, 2011; First review completed on February 14, 2011; accepted for publication on February 24 , 2011

E-mail: tanitsanchez@gmail.com

Tel.: 551130215251

\section{INTRODUCTION}

Tinnitus is the perception of sound in the absence of an acoustic external stimulus. ${ }^{1}$ Its prevalence is roughly estimated as being between $10 \%$ and $17 \%$ of the population ${ }^{2}$ and it afflicts around a third of North Americans beyond the age of $55 .^{3}$ For many years, tinnitus was thought to arise almost exclusively out of abnormal neuronal activity within the auditory pathways. However, accumulated evidence suggests that tinnitus-related neural activity is much more complex and multimodal than previously thought.

More often than ever, researchers are concluding that tinnitus can be evoked or modulated by inputs from the somatosensory, somatomotor and visual-motor systems in some individuals. ${ }^{4-16}$ This means that the psychoacoustic attributes of tinnitus (loudness and pitch) might be changed immediately -though only temporarily - by different stimuli, such as the following: forceful muscle contractions of head, neck and limbs; ${ }^{4-6}$ eye movements in the horizontal or vertical axis, ${ }^{7-9}$ pressure on myofascial trigger points; ${ }^{10,11}$ cutaneous stimulation of the hand/fingertip region ${ }^{12}$ and of the face; ${ }^{13}$ electrical stimulation of the median nerve and hand; ${ }^{14}$ or finger movements ${ }^{15}$ as well as orofacial movements. ${ }^{16}$

This specific subgroup is called somatosensory tinnitus and it seems to be a good example of central integration of the central nervous system, because an auditory symptom like tinnitus may be modulated immediately after various non-audiology stimuli are presented. The modulation phenomenon is yet to be fully understood, but there is

Copyright (c) 2011 CLINICS - This is an Open Access article distributed under the terms of the Creative Commons Attribution Non-Commercial License (http:// creativecommons.org/licenses/by-nc/3.0/) which permits unrestricted noncommercial use, distribution, and reproduction in any medium, provided the original work is properly cited. scientific evidence of existing neural connections between somatosensory and auditory systems, and their "activation" may play a role in this type of tinnitus. ${ }^{17,18}$

In clinical practice, tinnitus is still considered as an untreatable symptom, and many professionals tell patients that "there is nothing to be done" or that "you have to learn to live with it". Consequently, the practical value of broadening our knowledge of somatosensory tinnitus is that it might make it possible to use various treatment approaches involving a multidisciplinary team (otolaryngologists, physiotherapists and dentists). As a result, it is necessary to establish specific protocols for somatosensory tinnitus, for both its diagnosis and treatment.

\section{LITERATURE REVIEW}

\section{Tinnitus modulation}

Some authors have reported the existence of a connection between the proprioceptive and nociceptive afferents of the neck region as well as the cochlear nucleus - something that could explain ipsilateral correlation with tinnitus in cases of muscular tension. ${ }^{19,20}$ Furthermore, Levine ${ }^{21}$ suggested that somatic stimuli can disinhibit the ipsilateral cochlear nucleus, producing the excitatory neuronal activity within the auditory pathway that results in tinnitus.

The structure of the auditory pathway consists of several well-defined centers, although precise information about their interaction is still lacking. The cochlear nucleus is the first central nucleus of the auditory pathway, receiving information from the cochlear hair cells. In higher portions of the auditory pathway, the lemniscal system sends received information to the primary cortical auditory areas, whereas the extralemniscal portion of the ascending pathways transmits auditory information to associated areas. Many neurons of the extralemniscal system receive information from other sensorial tracts, such as the 
somatosensory system, which suggests an association between auditory pathways and non-auditory pathways. $^{22,23}$

According to Levine, ${ }^{21}$ tinnitus located ipsilateral in relation to the somatic injury raises suspicions regarding a possible somatosensory component in its origin. For Wright and Ryugo, ${ }^{17}$ the dorsal medullar nucleus, formed by cuneate and gracile nuclei, occupies a position in the somatosensory system similar to that of the cochlear nucleus in the auditory system. It receives information straight from the dorsal roots, which, in turn, receive information from the proprioceptive, tactile and vibratory receptors of the body surface. Therefore the lateral cuneate nucleus is the end-point of afferent fibers from the neck, ear and suboccipital muscles, and carries information about the head and ear position, which is needed in order to process the acoustic information.

Such theories relating to the anatomical relationship between both auditory and somatosensory pathways may help clarify the influence of myofascial pain on certain kinds of tinnitus as well as explain how myofascial trigger-point treatment could alleviate the symptom. Furthermore, such theories could also explain why certain patients with bilateral tinnitus reported relief in only one ear, corresponding with the side of the body with pain (or with the side of the body with the worst pain).

According to Shore et al., ${ }^{18}$ anatomical and physiological findings in animal studies have shown that the trigeminal and dorsal root ganglia relay some afferent somatosensory information from the periphery to secondary sensory neurons in the brain stem, specifically the spinal trigeminal nucleus and dorsal column nuclei, respectively. Each of these structures sends excitatory projections to the cochlear nucleus. Activation of the trigeminal ganglion elicits excitation in some dorsal cochlear nucleus units and inhibition in others. The modulation of firing rate and synchrony in dorsal cochlear nucleus neurons by somatosensory input is physiologically correlated with somatic tinnitus.

Among the theories attempting to explain modulation, neuroplasticity has been gaining positive recognition. Aberrant cross-modal plasticity seems to play a major role in tinnitus induced or modulated by somatosensory stimuli. ${ }^{24}$ This suggests that abnormal interactions between different sensory modalities, sensorimotor systems, neurocognitive and neuroemotional networks may contribute to certain aspects of tinnitus.

It is widely known that reorganization or re-mapping of specific central nervous areas occurs as a normal response of brain tissue to injury. ${ }^{25}$ However, like any double-edged sword, it is not possible to predict whether injury-induced plasticity will end up in limited or cross-modal effects, which in turn may result in compensatory or negative effects with pathological changes and unwanted clinical signs, as we have observed in some cases of tinnitus modulation. ${ }^{26}$ The effects of neural plasticity can generally be divided into early modifications and modifications with a later onset. ${ }^{25}$ The remodeling of tonotopic receptive fields within auditory structures (dorsal cochlear nucleus, inferior colliculus, and auditory cortex) seems to be a late manifestation of neural plasticity. Thus, modulation of tinnitus through stimulation of the somatosensory system might be explained by activation of auditory regions through the non-classical pathway. ${ }^{24}$

\section{Diagnosis of somatosensory tinnitus}

Somatosensory tinnitus is suspected clinically when anamnesis shows at least one of the following occurrences prior to the onset of tinnitus: (1) evident history of head or neck trauma, (2) tinnitus association with some manipulation of the teeth, jaw or cervical spine, (3) recurrent pain episodes in head, neck or shoulder girdle, (4) temporal coincidence of appearance or increase of both pain and tinnitus, (5) increase of tinnitus during inadequate postures during rest, walking, working or sleeping and (6) intense bruxism periods during the day or night.

This type of tinnitus often changes its loudness, pitch or localization during other stimulation coming from the head and neck. The most important characteristic of such tinnitus is that its origin seems to be related to problems of the head and neck, rather than to problems of the ear. Because of that, more than ever, these patients should be evaluated by an integrated team including an experienced dentist and a physiotherapist (or other professional) to evaluate possible bone or muscular disorders of the face, teeth and neck, in order to allow prompt diagnosis with a view to beginning adequate treatment as early as possible.

The phenomenon of somatosensory modulation of tinnitus (of any origin, with temporary changes in loudness, pitch or localization during somatosensory stimulation) may occur in patients with either otological or somatosensory tinnitus. As somatosensory modulation is very prevalent (65-80\% of patients with tinnitus), it is wise to search for this in all cases. Upon anamnesis, patients may spontaneously report that such temporary changes occur during common daily movements with the jaw or neck (opening of the mouth, clenching of the teeth or turning of the head) or by applying pressure on the temples, mandible, cheek, mastoid or neck with a fingertip. During physical evaluation, the professional actively searches for modulation through different stimuli, including digital pressure of myofascial trigger points, as well as asking patients to make active movements of the neck, shoulder, mandible and eyes (to detect gaze-evoked tinnitus). Because this is a short-lived effect, any questionnaire to evaluate tinnitus before and after the maneuver is difficult to use and a simple instrument, such as the visual analogy scale, is preferred to capture quick changes.

The audiogram is one of the basic complementary examinations that every patient with tinnitus should undergo. Diverse degrees of hearing loss are found in the vast majority of patients. The presence of symmetric pure tone thresholds in both ears (normal or abnormal) and unilateral or asymmetric tinnitus should direct the patient towards a more detailed evaluation of somatosensory tinnitus (besides excluding neural and central auditory diseases). One recent study showed that patients with normal hearing and chronic pain have greater improvement in their tinnitus when subjected to deactivation of myofascial trigger points. $^{27}$

Diagnosing somatosensory tinnitus and somatosensory modulation is not difficult. It depends mostly on wellconducted anamnesis and thorough physical examination. However, as most of the data referred to herein are recent, professionals need to incorporate the information into their daily routine so as to adequately diagnose such problems.

Somatosensory tinnitus is totally underdiagnosed due to the lack of publications on the subject. To date, there are no studies reporting sensitivity, specificity, diagnosis, odds 
ratio or receiver operator curves for any of the maneuvers described.

\section{Management of somatosensory tinnitus}

Somatosensory tinnitus patients need an evaluation of their temporomandibular joint and neck before the best treatment option can be selected. Otolaryngologists and neurologists are usually the first physicians to be sought by patients with tinnitus, but unfortunately little attention is given to the somatosensory influence on tinnitus. ${ }^{28}$ Many patients with tinnitus would benefit from the complementary opinion of a dentist or a physiotherapist (depending on their availability in each country). If a patient with tinnitus has muscular problems, such as tension in the temporomandibular joint or in the neck, these problems should be addressed. Various approaches for the treatment of tinnitus have been modified to include patients with somatosensory tinnitus.

\section{Relaxing muscle tension in the jaw and neck}

Because patients with temporomandibular disorder often present with muscular tension in both the jaw and neck - as well as tinnitus, vertigo/dizziness and aural fullness - the first aim of treatment for somatosensory tinnitus is the reduction of such muscular tension. ${ }^{29}$

Many patients with such problems also benefit from performing regular stretching exercises of their suboccipital muscles at home, as well as rotation movements in the atlanto-occipital joint - especially to the restricted side - and relaxing exercises involving breathing with the diaphragm.

Such treatment of muscle tension in the jaw and neck can reduce tension-related symptoms such as tinnitus, vertigo, aural fullness and pain in the jaw, neck or headache. Indeed, the intensity of all such symptoms was significantly reduced $(\mathrm{p}<0.001)$ after a 3-year follow-up examination for patients who used this type of treatment. ${ }^{29}$

Focal administration of lidocaine into jaw muscles (mainly the lateral pterygoid or the masseter) or into neck muscles (mainly the trapezius and the sternocleidomastoid) may temporarily reduce tension and, consequently, the tinnitus, while the local anesthetic is active. ${ }^{29}$

This means that relieving temporomandibular disorder and other forms of muscular tension may also relieve tinnitus. Felício et al. ${ }^{30}$ observed that patients with temporomandibular disorder and auditory symptoms improved after using bite splints for eight weeks. Moreover, Wright and Bifano ${ }^{31}$ reported an $82.5 \%$ improvement of tinnitus in patients whose temporomandibular disorders improved with the use of cognitive therapy, bite splints and home exercises.

\section{Manual therapies (cervical manipulation)}

Chiropractic care is a popular and successful management option for reversible functional disorders of the cervical spine and other body structures. Some studies have demonstrated that such manipulation can relieve tinnitus. ${ }^{32-36}$

Alcantara et al. ${ }^{34}$ described how chiropractic treatment could reduce tinnitus, vertigo and hearing loss in a patient with cervical subluxation and temporomandibular disorder. Symptoms eventually ceased after nine sessions. Kessinger et al. ${ }^{32}$ documented clinical changes after chiropractic sessions in a geriatric patient with tinnitus, vertigo, hearing loss and cervical alterations from C3 to C7. Throughout the sessions, the patient's symptoms were alleviated and structural/functional improvements were also evident through radiographic examination.

In contrast to classical chiropractic treatment, Arlen's atlas therapy is performed without traction, rotation or extension of the cervical spine. ${ }^{37}$ By means of irritation and tension of the posterior cervical muscle, one might increase the afferent input to the vestibular nuclei in the brain stem, which might give rise to tinnitus. ${ }^{37}$ Thus, reduction of the tension via atlas therapy seems to lower the proprioception and nociception output, leading to normalization of the flow of information to the brain stem, and, as a consequence, tinnitus improvement.

It seems that some somatosensory tinnitus could be alleviated by correcting the misalignment of the cervical spine through manual therapy (chiropractic or osteopathy), especially in the upper cervical region. Such readjustment might allow the entire spine to reposition itself and possibly readjust the input of the region through the somatosensory pathway on the auditory system.

According to Kaute, ${ }^{37}$ many other methods are used to relax the neck muscles, with some success in treating tinnitus: the Alexander method, autogenous training, the Brügger method, craniosacral treatment and Feldenkrais. All act on the same point - the posterior neck muscle.

Nevertheless, as much as this topic has been receiving more attention in the current literature, it still needs further clarification.

\section{Myofascial trigger point deactivation}

The relationship between myofascial trigger point (MTP) deactivation and tinnitus relief was initially demonstrated by injecting local anesthetic into these painful spots. In 1960, Travell $^{38}$ related tinnitus to MTPs located in the deep portion of the masseter muscle, because local injection of lidocaine on the ipsilateral masseter's myalgic spots relieved one patient's tinnitus. In this study, injections were repeated eight times and the relief lasted from several days up to four weeks. Wyant ${ }^{39}$ described two patients in whom tinnitus was relieved after injections with steroid and lidocaine into MTPs in the cervical region. In this study, both tinnitus and pain relief occurred for four months.

In an unpublished study of 178 patients who were compared with 39 subjects in a control group, tinnitus completely disappeared in $15 \%$ of the cases after cervical MTP injection of lidocaine $1 \%$. In a six-month follow-up after the last injection, tinnitus improved in more than $30 \%$ of the cases, as opposed to $15 \%$ from a control group. ${ }^{40}$

In 2005, Eriksson et al. ${ }^{41}$ showed that some tinnitus patients benefited from stretching and massage. Rocha and Sanche ${ }^{42}$ reported the case of a female patient who experienced improvement in her tinnitus, dizziness and chronic facial and cervical pain after having her MTP deactivated by pressure release and after having followed a home exercise program (muscle stretching, postural guidance and hot pack). In a double-blind placebo-control randomized clinical trial, we have shown a significant decrease in tinnitus loudness $(p<0.001)$, and in pain intensity $(p<0.001)$, a decrease in tinnitus handicap inventory scores $(p=0.01)$ and a decrease in the number of MTPs $(\mathrm{p}<0.001)$ from MTP inactivation by pressure release. ${ }^{43} \mathrm{We}$ consider pressure release as a gradual and sustained digital pressure on the MTP until the patient no longer perceives any sensory abnormality, referred pain or discomfort at the spot where such pressure is applied. 
The treatment was based on 10 sessions of real MTP deactivation and 10 sessions of placebo MTP deactivation in tinnitus patients. The placebo part of the control-group session related to a fake treatment, that is, pressure applied to points adjacent to each real MTP and not to the real MTP. The real treatment used pressure release in each MTP of eight possible muscles (infraspinatus, levator scapulae, superior trapezius, splenius capitis, splenius cervicis, sternocleidomastoid, masseter and temporalis) along with home orientations (muscle stretching, postural guidance and hot pack). Thirty-seven patients from the experimental group and 34 patients from the control group were analyzed blind by a researcher before treatment began and again after 10 weeks of treatment. Ultimately, three patients with unilateral tinnitus benefitted from complete relief, two patients with bilateral tinnitus felt complete relief in one of the ears, and one patient reported an improvement in the number of perceived sounds (a decrease from seven to three). All of these patients maintained their relief following the two-month revaluation. Patients with normal pure tone audiograms were the ones with the best results after treatment, regardless of whether the tinnitus was constant or intermittent, unilateral or bilateral. Another remarkable finding was that patients in whom tinnitus intensity had diminished during modulation had better results during treatment when compared with those in whom tinnitus intensity had increased.

Many techniques dealing with MTP relief have been published, but few have been supported by scientific evidence. The most commonly used treatment procedures for myofascial pain syndrome were reviewed by Vernon and Schneider, ${ }^{44}$ showing that they were supported by evidence. Laser therapy, pressure release, transcutaneous electrical nerve stimulation, acupuncture and magnet therapy for MTP and myofascial pain syndrome are treatments that are scientifically supported to varying degrees. The duration of relief fluctuates across the therapies. Evidence is weak, however, for ultrasound therapy, as it is also for electrical muscle stimulation, high-voltage galvanic stimulation, interferential current and frequency modulated neural stimulation.

\section{Training exercises repeating movements that evoke tinnitus modulation}

Exercise in general may be beneficial because it increases brain-derived neurotrophic factor. ${ }^{45}$ Training with repetitive movements generates specific neurophysiological changes through the activation of neural plasticity. Activation of neural plasticity has been proved to have therapeutic effects on many disorders, such as vestibular diseases, where the repetition of specific maneuvers can decrease vestibular disturbances. Sanchez et al. ${ }^{46}$ showed that muscle contractions may change the pattern of tinnitus, from temporary worsening to temporary improvement, with repetition of the maneuvers that modulate tinnitus. A similar strategy was able to cure one of our patients, who had gaze-evoked tinnitus, at the Tinnitus Research Group of the University of São Paulo School of Medicine, by repeating all the eye maneuvers that evoked her tinnitus. ${ }^{26,47}$ The central element of vestibular rehabilitation is the repetition of a set of exercises, which compensates for the central nervous system abnormalities using eye movements, as well as cervical and body maneuvers. In this case, the disappearance of gaze-evoked tinnitus occurred with the repetition of eye movements that used to evoke it. The vertical component of the patient's gaze-evoked tinnitus responded sooner to treatment than the horizontal component, which indicates that more than one neural network or process is involved in this process.

Thus, individuals with somatosensory tinnitus or tinnitus that can be modulated may experience relief through coordinated exercise of the muscles that can modulate tinnitus. Future studies may add further information.

\section{Transcutaneous nerve stimulation}

The effect of transcutaneous nerve stimulation is based on its ability to induce widespread, non-segmental and prolonged increased microcirculation due to simpaticoinhibition in vascular beds. ${ }^{48}$ Kaada et al. ${ }^{48}$ treated 29 tinnitus patients by using transcutaneous nerve stimulation: nine patients reported reduction in their tinnitus. The improvement was more common with low-frequency (125-500 Hz) tinnitus. Seven patients also reported improvements in hearing, mostly in the low-frequency band. In 1997, Rahko and Kotti ${ }^{49}$ treated 26 tinnitus patients with transcutaneous nerve stimulation. Except for three patients with normal hearing, all had cochlear hearing losses. Tinnitus disappeared in none of them, but was diminished in 7 cases, as opposed to 3 out of 24 non-treated control subjects. Among the controls there was no relief in lowfrequency tinnitus cases. In the Aydemir et al. ${ }^{50}$ study, the efficacy of transcutaneous nerve stimulation for the management of tinnitus symptoms was evaluated in 22 patients. The authors concluded that it is a useful method for improving the quality of life of patients with tinnitus.

\section{Hypnosis}

Attias et al. ${ }^{51}$ compared self-hypnosis, masking and "attentiveness to the patient's complaints" in the treatment of tinnitus. Self-hypnosis reduced tinnitus significantly, attentiveness reduced it partly, and masking did not have any significant effect. Marks et al. ${ }^{52}$ tried hypnotherapy for chronic tinnitus. One out of 14 patients showed a highly significant response to the latter treatment, as judged by visual analogue scales. Five out of 14 patients (36\%) found the induction of a hypnotic state to be worthwhile. It seemed to help them tolerate their tinnitus better, although its loudness and quality were unaltered. On the other hand, Coles and Hallam ${ }^{53}$ tried hypnosis and relaxation with two tinnitus patients. One of them was given a post-hypnotic suggestion that whenever he was aware of tinnitus it changed to pleasant music. Tinnitus was relieved in both patients. In another experiment the combination of relaxation and hypnotherapy was used to change tinnitus to a more pleasant sound. Both patients were also taught to "turn tinnitus down" with an imaginary control knob. After treatment, tinnitus was better accepted by the patients than before it.

\section{Botulinum toxin type $A$}

Botulinum toxin type A (Bot-A) is a neurotoxin that can inhibit the release of acetylcholine at the neuromuscular junction. $^{54}$ Because of its known paralytic effect, it is administered locally to control muscle hyperactivity in many different disorders, as well as in cosmetic procedures.

Besides the paralytic effect, Bot-A might have direct antinociceptive action through blockage of the autonomic nervous system in addition to neuromuscular action. ${ }^{55-59}$ 
Moreover, through a peripheral mechanism it can inhibit the sensitization of central trigeminovascular neurons. ${ }^{60}$ Thus, Bot-A has more than one effect with regard to headache, migraine and chronic neuropathic pain control. Recently, Bot-A has been used for the treatment of tinnitus. $^{61,62}$

A crossover double-blind study of the effect of Bot-A showed that it had little effect on tinnitus. ${ }^{62}$ One group was first injected with Bot-A and four months later with saline injections into three sites around the ear: $1 \mathrm{~cm}$ above the pinna, $1 \mathrm{~cm}$ behind (at a "2 o'clock" position), and $1 \mathrm{~cm}$ behind auricle (at a "5 o'clock" position). The second group was first injected with a placebo and, four months later, with Bot-A. Seven participants reported a decrease in their tinnitus after the Bot-A injection, and two improved after the placebo.

When tinnitus was analyzed through the global clinical impression of the patient (i.e. "better", "worse" or "the same"), the effects of Bot-A were significantly better than the placebo $(p<0.05)$, when comparing pre-treatment and 4 months after injection $(p=0.04)$. Such results suggest that the Bot-A can play a role in tinnitus management by reducing peripheral inputs from the cervical, temporal, frontal and periauricular muscles.

A similar study showed that, out of 26 participants, 7 improved, 13 worsened and 16 were unchanged. ${ }^{61}$ When treatment with the botulinum toxin is being considered, potentially serious side-effects, such as changing cardiac reflexes, should be considered. ${ }^{63}$ Eighty percent of people treated with the botulinum toxin reported abnormalities in their electrocardiograms. The fact that the treatment is likely to demand repetition for long periods is a disadvantage that - when used for the treatment of other disorders such as hemifacial spasm - has made many people discontinue treatment. ${ }^{64}$

\section{CONCLUSION}

Studies have been pointing towards a high prevalence of somatosensory tinnitus among tinnitus patients. Thus, professionals involved with providing treatment for patients with tinnitus must differentiate this specific subgroup from the conventional ones. Investigating the presence of somatosensory tinnitus by means of a specific protocol and choosing adequate therapeutic options that will target auditory pathways and musculoskeletal disorders may increase the possibility of satisfactory results.

\section{REFERENCES}

1. Jastreboff PJ, Sasaki CT. An animal model of tinnitus: a decade of development. Am J Otol. 1994;15:9-11.

2. Seidman MD, Jacobson GP. Update on tinnitus. Otolaryngol Clin North Am. 1996;29:455-65

3. Lockwood AH, Salvi RJ, Burkard RF, Galantowicz PJ, Coad ML, Wack DS. Neuroanatomy of tinnitus. Scand Audiol. 1999;28:47-52, doi: 10. 1080/010503999424905.

4. Levine RA. Somatic Modulation appears to be a fundamental attribute of tinntus. In: Hazell JPW, editor. Proceedings of the Sixth International Tinnitus Seminar. London: The Tinnitus and Hyperacusis Center; 1999. pp. 193-7.

5. Sanchez TG, Guerra GCY, Lorenzi MC, Brandão AL, Bento RF. The influence of voluntary muscle contractions upon the onset and modulation of tinnitus. Audiol Neurootol. 2002;7:370-5, doi: 10.1159/ 000066155.

6. Sanchez TG, Lima AS, Brandao AL, Lorenzi MC, Bento RF. Somatic modulation of tinnitus: test reliability and results after repetitive muscle contraction training. Ann Otol. 2007;116:30-5.
7. Coad ML, Lockwood A, Salvi R, Burkard R. Characteristics of patients with gaze-evoked tinnitus. Otol Neurotol. 2001;22:650-4, doi: 10.1097/ 00129492-200109000-00016.

8. Baguley DM, Phillips J, Humphriss RL, Jones S, Axon PR, Moffat DA. The prevalence and onset of gaze modulation of tinnitus and increased sensitivity to noise after translabyrinthine vestibular schwannoma excision. Otol Neurotol. 2006;27:220-4, doi: 10.1097/01.mao.0000172412. 87778.28.

9. Sanchez TG, Pio MRB. Abolição de zumbido evocado pela movimentação ocular por meio de repetição do deslocamento do olhar: um método inovador. Arq Otorhinolaryngol. 2007;11:451-3.

10. Rocha CACB, Sanchez TG. Myofascial trigger points: another way of modulating tinnitus. Prog Brain Res. 2007;166:209-14, doi: 10.1016/ S0079-6123(07)66018-X.

11. Rocha CACB, Sanchez TG, Siqueira JTT. Myofascial trigger points: a possible way of modulating tinnitus? Audiol Neurootol. 2008;13:153-60, doi: $10.1159 / 000112423$.

12. Cacace AT, Cousins JP, Parnes SM, McFarland DJ, Semenoff D, Holmes T, et al. Cutaneous-evoked tinnitus. II. Review of neuroanatomical, physiological and functional imaging studies. Audiol Neurootol. 1999;4:247-57, doi: 10.1159/000013848.

13. Sanchez TG, Marcondes RA, Kii MA, Lima AS, Rocha CAB, Ono CR, et al. A different case of tinnitus modulation by tactile stimuli in a patient with pulsatile tinnitus. Presented at the Second Meeting of the Tinnitus Research Initiative, Monaco, 2007. pp. 21-3.

14. Møller AR, Rollins PR. The non-classical auditory pathways are involved in hearing in children but not in adults. Neurosci Lett. 2002;319:41-4, doi: 10.1016/S0304-3940(01)02516-2.

15. Cullington $\mathrm{H}$. Tinnitus evoked by finger movement: brain plasticity after peripheral deafferentation. Neurology. 2001;56:978.

16. Pinchoff RJ, Burkard RF, Salvi RJ, Coad ML, Lockwood AH. Modulation of tinnitus by voluntary jaw movements. Am J Otol. 1998;19:785-9.

17. Wright DD, Ryugo DK. Mossy fiber projections from the cuneate nucleus to the cochlear nucleus in the rat. J Comp Neurol. 1996;365:159-72, doi: 10.1002/(SICI)1096-9861(19960129)365:1<159::AID-CNE12>3.0.CO;2-L.

18. Shore S, Zhou J, Koehler S. Neural mechanisms underlying somatic tinnitus. Prog Brain Res. 2007;166:107-23, doi: 10.1016/S00796123(07)66010-5.

19. Young ED, Nelken I, Conley RA. Somatosensory effects on neurons in dorsal cochlear nucleus. J Neurophysiol. 1995;73:743-65.

20. Wright DD, Ryugo DK. Mossy fiber projections from the cuneate nucleus to the cochlear nucleus in the rat. J Comp Neurol. 1996;365:159-72, doi: 10.1002/(SICI)1096-9861(19960129)365:1<159::AID-CNE12>3.0.CO;2-L.

21. Levine RA. Somatic (craniocervical) tinnitus and the dorsal cochlear nucleus hypothesis. Am J Otolaryngol. 1999;20:351-62, doi: 10.1016/ S0196-0709(99)90074-1.

22. Aitkin LM, Irvine DR, Nelson JE, Merzenich MM, Clarey JC. Frequency representation in the auditory midbrain and forebrain of a marsupial, the northern native cat (Dasyurus hallucatus). Brain Behav Evol. 1986;29:1728, doi: $10.1159 / 000118669$.

23. Møller AR, Møller MB, Yokota M. Some forms of tinnitus may involve the extralemniscal auditory pathway. Laryngoscope. 1992;102:1165-71, doi: 10.1288/00005537-199210000-00012.

24. Cacace AT. Expanding the biological basis of tinnitus: crossmodal origins and the role of neuroplasticity. Hear Res. 2003;175:112-32, doi: 10. 1016/S0378-5955(02)00717-7.

25. Chen R, Cohen LG, Hallett M. Nervous system reorganization following injury. Neuroscience. 2002;111:761-73, doi: 10.1016/S0306-4522(02)000258.

26. Sanchez TG, Kii MA. Modulating tinnitus with visual, muscular and tactile stimulation. Seminars in Hearing, Tinnitus: part II. 2008;29:350-60.

27. Sanchez TG, Rocha CACB. Patients with normal hearing and chronic pain experience more relief from tinnitus when subject to deactivation of myofascial trigger points. In: XXXI International Congress of Audiology 2010, São Paulo.

28. Sanchez TG. What to expect from your physician and other healthcare professionals. In: Tyler R, editor. The Consumer Handbook on Tinnitus. Sedona: Auricle Ink Publishers; 2008. p. 183.

29. Björne A. Assessment of temporomandibular and cervical spine disorders in tinnitus patients. Prog Brain Res. 2007;166:215-9, doi: 10. 1016/S0079-6123(07)66019-1.

30. Felício CM, Melchior MO, Ferreira CL, Silva MA. Otologic symptoms of temporomandibular disorder and effect of orofacial myofunctional therapy. Cranio. 2008;26:118-25.

31. Wright EF, Bifano SL. Tinnitus improvement through TMD therapy. J Am Dent Assoc. 1997;128:1424-32.

32. Kessinger RC, Boneva DV. Vertigo, tinnitus, and hearing loss in the geriatric patient. J Manipulative Physiol Ther. 2000;23:352-62.

33. Kessinger RC, Boneva DV. Case study: acceleration/deceleration injury with angular kyphosis. J Manipulative Physiol Ther. 2000;23:279-87, doi: 10.1016/S0161-4754(00)90175-1.

34. Alcantara J, Plaugher G, Klemp DD, Salem C. Chiropractic care of a patient with temporomandibular disorder and atlas subluxation. J Manipulative Physiol Ther. 2002;25:63-70, doi: 10.1067/mmt.2002.120415. 
35. Leboeuf-Yde C, Pedersen EN, Bryner P, Cosman D, Hayek R, Meeker WC, et al. Self-reported nonmusculoskeletal responses to chiropractic intervention: a multination survey. J Manipulative Physiol Ther. 2005;28:294-302, doi: 10.1016/j.jmpt.2005.04.010.

36. DeVocht JW, Schaeffer W, Lawrence DJ. Chiropractic treatment of temporomandibular disorders using the activator adjusting instrument and protocol. Altern Ther Health Med. 2005;11:70-3.

37. Kaute BB. The influence of Atlas therapy on tinnitus. Int Tinnitus J. 1998;4:165-7.

38. Travell J. Temporomandibular joint pain referred from muscle of the head and neck. J Prosthet Dent. 1960;10:745-63, doi: 10.1016/00223913(60)90257-2.

39. Wyant GM: Chronic pain syndromes and their treatment. II. Trigger points. Canad Anaesth Soc J. 1979;26:216-9, doi: 10.1007/BF03006985.

40. Estola-Partanen M. Muscular tension and tinnitus: an experimental trial of trigger point injections on tinnitus (dissertation). Tampere, University of Tampere, 2000

41. Eriksson M, Gustafsson S, Axelsson A. Tinnitus and trigger points: a randomized cross-over study. In: Reich GE, Vernon JA, editors. Proceedings of the Fifth International Tinnitus Seminar. Portland, 1995. pp. 81-3.

42. Rocha CACB, Sanchez TG. Tinnitus modulation by miofascial trigger points and its disappearance by treatment of the myofascial pain syndrome: an interesting result. In: Langguth B. Abstracts of the Second Meeting of Tinnitus Research Initiative, Monaco, 2007. Univ. of Regensburg, 2007, p. 49.

43. Rocha CACB. Eficácia da Desativação dos Pontos-gatilho Miofasciais para o Tratamento do Zumbido em Pacientes com Síndrome Dolorosa Miofascial. São Paulo: Universidade de São Paulo; 2010.

44. Vernon H, Schneider M. Chiropractic management of myofascial trigger points and myofascial pain syndrome: a systematic review of the literature. J Manipulative Physiol Ther. 2009;32:14-24, doi: 10.1016/j. jmpt.2008.06.012.

45. Vaynman S, Gomez-Pinilla F. License to run: exercise impacts functional plasticity in the intact and injured CNS by using neurotrophins. Neurorehabil Neural Repair. 2005;19:283-95, doi: 10.1177/ 1545968305280753.

46. Sanchez TG, Lima AS, Brandão AL, Lorenzi MC, Bento RF. Somatic modulation of tinnitus: test reliability and results after repetitive muscle contraction training. Ann Otol Rhinol Laryngol. 2007;116:30-5.

47. Sanchez TG, Pio MRB. Abolição de zumbido evocado pela movimentação ocular por meio da repetição do deslocamento do olhar: um método inovador. Arq Int Otorrinolaringol. 2007;11:345-9.

48. Kaada B, Hognestad S, Havstad J. Transcutaneous nerve stimulation (TNS) in tinnitus. Scand Audiol. 1989;18:211-7, doi: 10.3109/ 01050398909042196.

49. Rahko T, Kotti V. Tinnitus treatment by transcutaneous nerve stimulation (TNS). Acta Otolaryngol Suppl. 1997;529:88-9, doi: 10.3109/ 00016489709124091.
50. Aydemir G, Tezer MS, Borman P, Bodur H, Unal A. Treatment of tinnitus with transcutaneous electrical nerve stimulation improves patients' quality of life. J Laryngol Otol. 2006;120:442-5, doi: 10.1017/ S0022215106000910.

51. Attias J, Shemesh Z, Sohmer H, Gold S, Shoham C, Faraggi D. A comparison between self-hypnosis, masking and attentiveness for alleviation of chronic tinnitus. Audiology. 1993;32:205-12, doi: 10.3109/ 00206099309072936.

52. Marks NJ, Karl H, Onisiphorou CA. controlled trial of hypnotherapy in tinnitus. Clin Otolaryngol Allied Sci. 1985;10:43-6, doi: 10.1111/j.13652273.1985.tb01163.x.

53. Coles RRA, Hallam RS. Tinnitus and its management. Br Med Bull. 1987;43:983-98.

54. Simpson LL. The origin, structure, and pharmacological activity of botulinum toxin. Pharmacol Rev. 1981;33:155-88.

55. Blersch W, Schulte-Mattler WJ, Przywara S, May A, Bigalke H, Wohlfarth K. Botulinum toxin $\mathrm{A}$ and the cutaneous nociception in humans: a prospective, double-blind, placebo-controlled, randomized study. J Neurol Sci. 2002;205:59-63, doi: 10.1016/S0022-510X(02)00313-1.

56. Dolly O. Synaptic transmission: inhibition of neurotransmitter release by botulinum toxins. Headache. 2003;43:16-24, doi: 10.1046/j.1526-4610.43. 7s.4.x.

57. Aoki KR. Review of a proposed mechanism for the antinociceptive action of botulinum toxin type A. Neurotoxicology. 2005;26:785-93, doi: 10 1016/j.neuro.2005.01.017

58. Cui M, Khanijou S, Rubino J, Aoki KR. Subcutaneous administration of botulinum toxin A reduces formalin-induced pain. Pain. 2004;107:12533, doi: 10.1016/j.pain.2003.10.008.

59. Durham PL, Cady R. Regulation of calcitonin gene-related peptide secretion from trigeminal nerve cells by botulinum toxin type A: implications for migraine therapy. Headache. 2004;44:35-42, doi: 10. 1111/j.1526-4610.2004.04007.x.

60. Oshinsky M, Poso-Rosich P, Luo J, Hyman S, Silberstein SD. Botulinum toxin A blocks sensitization of neurons in the trigeminal nucleus caudalis. Cephalalgia. 2004;24:781

61. Stidham KR, Solomon PH, Roberson JB. Evaluation of botulinum toxin A in treatment of tinnitus. Otolaryngol Head Neck Surg. 2005;132:883-9, doi: 10.1016/j.otohns.2005.02.015.

62. Láinez MJ, Piera A. Botulinum toxin for the treatment of somatic tinnitus. Prog Brain Res. 2007;166:335-8, doi: 10.1016/S00796123(07)66031-2.

63. Girlanda P, Vita G, Nicolosi C, Milone S, Messina C. Botulinum toxin therapy: distant effects on neuromuscular transmission and autonomic nervous system. J Neurol Neurosurg Psychiatry. 1992;55:844-5, doi: 10. 1136/jnnp.55.9.844

64. Marion MH. Hemifacial spasm: treatment with botulinum toxin (long term results). In: Sindou M, Keravel Y, Møller AR. Hemifacial Spasm. A Multidisiplinary Approach. Springer: Wien; 1997. pp. 141-4. 